\title{
Modeling the process of removal aimed at cut traces on semiconductor wafers by using the method of contactless chemical-and-dynamical polishing
}

\author{
G.A. Pashchenko, M.Yu. Kravetskyi, A.V. Fomin \\ V. Lashkaryov Institute of Semiconductor Physics, NAS of Ukraine, 03028 Kyiv, Ukraine \\ E-mail:ipsfomin@ukr.net
}

\begin{abstract}
Used in this work is the stationary model of the process of chemical-anddynamical polishing (CDP) the substrates in the case of balance between diffusion, convective and chemical fluxes. Obtained has been an analytical expression relating the surface shape in processed material with physical parameters of processes taking place under CDP. Calculations performed by the authors enabled to find technological regimes of processing that provides total removal of linear morphological defects from the surface of substrates after cutting. Comparison of experimental profilograms taken from the processed surfaces with theoretical dependences showed their satisfactory agreement.
\end{abstract}

Keywords: substrate, surface, chemical-and-dynamical polishing, cutting.

Manuscript received 11.03.15; revised version received 18.06.15; accepted for publication 03.09.15; published online 30.09.15.

\section{Introduction}

The process of high-quality polishing the surface of semiconductor wafers (substrates) is an intrinsic stage in technologies aimed at production of micro-electronics objects. Application of abrasive methods in operations for cutting the ingot of semiconductor material by wafers, for grinding and polishing the wafer surface, which provides removal of cut traces (cut strips, scratches, spallings) and thinning the wafers inevitably leads to creation of a structurally destructed surface layer of a definite depth [1-6].

Removing it is a complex technological task and, as a rule, is realized using the methods of chemical forming the surface, i.e., applying the operations of chemical-and-mechanical polishing (CMP) or chemicaland-dynamical polishing (CDP), which needs developing optimal technological regimes and compositions of polishing etchants $[1,2,7,8]$.
Offered in the works $[9,10]$ is the operation of nonabrasive contactless chemical-and-mechanical polishing (NCMP) the substrates and developed its physical model. The difference between the NCMP operation and CMP/CDP operations is that the processed sample surface has no contact with the polisher, because of the substrate is slightly raised above the surface of the rotating polisher due to the flotation force appearing in the course of movement. Thus, convective-and-diffusion processes of polishing take place in a narrow gap (approximately $10 \mu \mathrm{m}$ ) filled with etchant, what differ them from those under free etching. Therefore, the main advantage of the NCMP operation lie in the possibility to obtain surfaces without introduction of a structurally-destructed surface layer as well as remove traces of linear morphological imperfections from these surfaces due to chosing the necessary technological regimes avoiding intermediate abrasive grinding and polishing. 


\section{Experimental details}

To model the process of NCMP removing the morphological defects (scratches, traces of slicing) that occur on substrate surface after abrasive cutting them from an ingot, we performed a number of experiments.

Preliminary, we prepared the samples of three types, namely:

1) using the wafers with the surface grinded applying a thin polishing powder;

2) after polishing the surface by using the NCMP method with a hard plexiglass polisher for the time period sufficient to reach a stationary shape [9];

3) with the surface of the stationary shape after polishing the substrates by using the NCMP method with elastomer.

On the surface of substrates processed in the above ways, using a diamond cutter we made scratches of various depths oriented along the movement direction of polishing etchant. Then, the prepared samples were polished using the NCMP method in different processing regimes, and after every polishing cycle we checked the scratch shape as well as the thickness of the layer removed from the sample surface.

Dynamics of changing the scratch depth and width was studied by recording and analysis of profilograms inherent to the sample surface after each polishing cycle (we used the profilograph-profilometer of the firm "Calibr", the series 252). The thickness of the removed layer was determined in five points over the wafer surface.

To study dynamics of removing the morphological defects, we used the model material - wafers of monocrystalline $\mathrm{NaCl}$ of dimensions $2 \times 1 \mathrm{~cm}^{2}$; as etchant, the solution of water in ethanol with the concentration $0.2 \mathrm{~g} / \mathrm{ml}$ was used. The velocity of the polisher movement was close to $7 \mathrm{~cm} / \mathrm{s}$.

\section{Results and discussion}

For using the nonabrasive contactless chemical-andmechanical processing the wafer surfaces, we developed a convective-diffusion model of processes taking place under this operation, which enabled us to mathematically describe the wafer surface shape and the velocity of removing the surface layer (polishing rate) for the case when all the processes become stationary, i.e., all the points of the polished surface are etched with the same velocity [9]. As it follows from the model and profilograms obtained in experiments, the shape of the stationary surface is the plane inclined relatively to the polisher plane under the angle $B$, and has the edge shape described by the exponent of the power $k$. The stationary surface can be expressed as follows:

$y_{S}(x)=A-B x+E e^{-k x}$, where $A=2 \delta+\frac{D \cdot l}{U \delta}, B=\frac{D}{U \delta}$,

$E=\frac{2 \delta}{\pi^{2}}, k=-\frac{D \pi^{2}}{U \delta^{2}}$.
Here, $\delta$ is the halfwidth of the gap between the sample and polisher filled with etchant, $D$ - diffusion coefficient of the active component in etchant, $U-$ velocity of polisher movement, $l$-sample length. As it follows from (1), the $B$ and $k$ coefficients determining the surface geometry depend on technological parameters of the poilishing process.

The formula for the stationary velocity of surface polishing $V$ can be obtained as follows:

$\frac{1}{V}=\frac{\rho l}{U \delta \beta C_{0}}+\frac{\rho}{\alpha \beta C_{0}}+\frac{\rho \delta}{D \beta C_{0}}$,

where $\rho$ is the density of the sample substance, $C_{0}-$ concentration of the etchant active component, $\alpha-$ constant inherent to this chemical reaction, $\beta$ - amount of the wafer substance solved in the unit mass of the etchant active component. The expression (2) is the equation of the second power relatively to $\delta$, and, thereof, the function $V(\delta)$ has its extremum in the point

$\delta_{m}=\sqrt{\frac{l D}{U}}$

Shown in Fig. 1 in accord with (2), is the dependence for the polishing velocity $V$ at the sample surface on the $\delta$ value for four linear velocities of the polisher movement as well as on $\rho, C_{0}, \alpha, \beta$ and $U$ values corresponding to these experimental conditions. The point $\delta_{m}$ shifts in accord with the formula (3) to the side of lower $\delta$ values with increasing the polisher velocity (Fig. 1a).

The non-linear dependence of the etching velocity at the wafer surface results in some features related with "curing" the scratches. Indeed, if the regime of polishing has such parameters (when etching the scratch of the depth $h$ ) that the thickness of the etchant layer between the polisher surface and scratch bottom is $\left(\delta_{\text {oper }}+h\right)$ and $\left(\delta_{\text {oper }}+h\right) \leq \delta_{m}$, then polishing takes place on the left side relatively $\delta_{m}$, where the etching velocity is in proportion to the $\delta$ value.

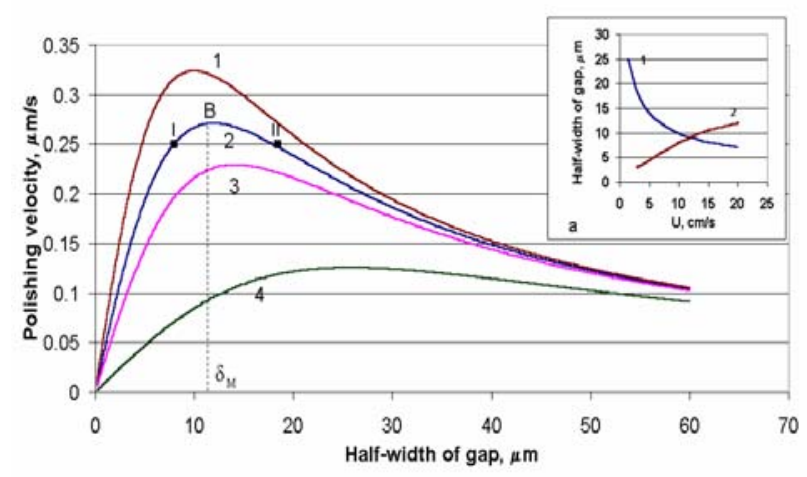

Fig. 1. Dependence of the polishing velocity on the etchant thickness $\delta$ for the polisher movement speeds: $1-10 ; 2-7$; $3-5$; and $4-1.5 \mathrm{~cm} / \mathrm{s}$. Shown in the inset (a) are the dependences of $\delta_{m}(1)$ and experimentally found $\delta_{\text {oper }}(2)$ values on the polisher movement speed. 
Therefore, the scratch bottom (which corresponds to the point $\left(\delta_{\text {oper }}+h\right)$ ) will be etched with a higher velocity than the wafer surface (corresponding to the $\delta_{\text {oper }}$ point), and the scratch will be etched and deepened in the polishing process, i.e., it is the range of non-stable polishing. In the case when $\delta_{\text {oper }} \geq \delta_{m}$, the point $\left(\delta_{\text {oper }}+h\right)$ is etched with the lower velocity than that in the point $\delta_{\text {oper, }}$, then the scratch is "cured". It corresponds to the range of stable polishing, when the depth of the scratch is lowered.

Let us consider the experimental results. Fig. 2 shows changes in the depth of longitudinal scratches under stage-by-stage contactless chemical-anddynamical polishing. $\mathrm{X}$-axis corresponds to the value of thickness typical for the layer $d$ removed from the wafer surface for the corresponding time. It enables to observe the relation between the velocities of etching at the scratch bottom and at the wafer surface.

As follows from the figure, in all the cases together with etching the sample surface there takes place etching of the scratch bottom, but the velocity of the latter process for the curves 1 to 3 is lower than that of the former. It is obvious that, when curing the scratch, it is necessary to remove from the wafer surface a layer, the thickness of which is higher than the scratch depth. In our case, in conditions of forming the scratches on the stationary surface this ratio was $\geq 2$ (curve 1 ).

In the second case, when scratches were formed on the non-stationary surface (curve 2), the ratio between the above values becomes higher, since at the initial stage (when the stationary regime is being reached) curing the scratch goes slower, and transition of the processes to the stationary level corresponds to the inflection point in the curve 2. Under polishing with elastomer (curve 3), the distance between the polisher surface and the crystal one $\delta_{\text {oper }}$ was increased, which resulted in growth of the difference between polishing velocities for the scratch bottom and wafer surface and, respectively, to faster curing the scratches under other equal conditions.

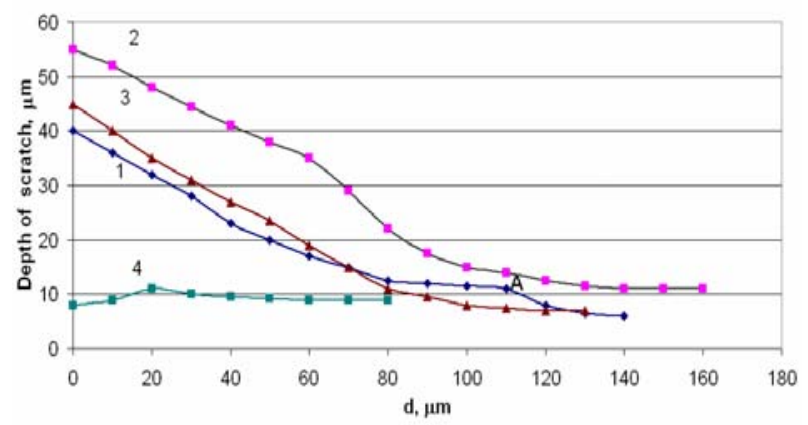

Fig. 2. Dependence of the depth inherent to longitudinal scratches on the thickness of the layer removed from the wafer surface in the course of polishing for the cases of scratch formation: 1, 4-on the stationary surface; $2-$ on the grinded (non-stationary) surface; 3 - on the stationary surface under polishing with application of elastomer.
Besides, as seen from Fig. 2 (curves 1 and 2), the scratch depth is decreased to the definite critical value $h_{\text {cr }}$ (approximately $10 \mu \mathrm{m}$ in this case) that is practically unchanged in the process of further polishing, and curing takes place due to widening the scratch. For the curve 3 corresponding to polishing with elastomer, $h_{\mathrm{cr}}$ is lower and close to approximately $6 \mu \mathrm{m}$. This value can be decreased using the increased rotation speed of the polisher $U$, which can be seen in the curve 1 . Here, the increase of $U$ from $7 \mathrm{~cm} / \mathrm{s}$ up to $10 \mathrm{~cm} / \mathrm{s}$ in the point $A$ results in the lowered $h_{\mathrm{cr}}$ value down to $7 \mu \mathrm{m}$.

This character of the $h(d)$ dependence is in full accordance with the theoretical conceptions about the NCMP process (Fig. 1). The polishing velocity on the wafer when realizing the process with a smooth polisher without elastomer was approximately $24 \mu \mathrm{m} / \mathrm{min}$, while the halfwidth of the etchant layer $\delta$ calculated from the slope of the plane of stationary surface relatively to the polisher plane $B$ (Eq. (1)) was close to $7 \mu \mathrm{m}$. Thus, processing the sample surface takes place in the range of unstable polishing (Fig. 1, point I). The distances of the scratch bottom to the polisher plane $\delta=\left(\delta_{\text {oper }}+h\right)$ for the curves 1,2 and 3 (Fig. 2) are close to 47, 59 and $52 \mu \mathrm{m}$, respectively, and correspond to the range of stable polishing up to the moment when the etching velocities for the wafer surface and scratch bottom become equal (Fig. 1, point II), and $h$ remains constant during further polishing.

The curve 4 in Fig. 2 corresponds to the case when the value $\left(\delta_{\text {oper }}+h\right)$ lies in the range of instability. Therefore, this scratch at the first polishing stage is deepened up to the value $\left(\delta_{\text {oper }}+h\right)=\delta_{m}$, and its depth can be decreased with increasing $U$.

Thus, total curing the scratches is related with increasing the rotation speed of the polisher. Shown in Fig. 1a are the dependences of $\delta_{m}$ and $\delta_{\text {oper }}$ on the polisher rotation speed (dependence of $\delta_{\text {oper }}$ on $U$ was found from experiments). The cross point of these curves enables to estimate the minimal polisher rotation speed $U_{\min }$, when there realized is the condition $\delta_{\text {oper }}=\delta_{m}$. If polishing is fulfilled at the velocities $U>U_{\min }$, the operation point goes to the stable branch of polishing, and in conditions of NCMP the scratches of arbitrary depths are fully polished out.

\section{Conclusions}

It has been proved experimentally that, to remove longitudinal scratches by using the method of contactless chemical-and-dynamical polishing, it is necessary to etch out from the wafer surface the layer approximately three-fold exceeding the defect depth.

In accord with calculations based on the offered convection-diffusion model for nonabrasive chemicaland-mechanical polishing, the total removal of scratches is possible by chosing such polishing regime that the etchant layer thickness between surfaces of the polisher 
and wafer exceeds the $\delta_{m}$ value determined from the relation (3), which is provided by chosing the polisher rotation speed satisfying the condition $U>U_{\min }$.

Application of the NCMP method in the process of preparing the semiconductor wafers (substrates) allows avoiding the intermediate stages of abrasive grinding and polishing, if the initial wafer need not considerable thinning.

\section{References}

1. Keohra Sangwal, Etching of Crystals. Theory, Experiment and Application. Moscow, Mir, 1990.

2. V.A. Perevoshchikov, Processes of chemical-anddynamical polishing the semiconductor surface // Vysokochistye veshchestva, No 2, p. 5-29 (1995), in Russian.

3. United States Patent $8844511 ; 30.09 .14$. Current Intern. Class: B28D 1/06. Method for slicing a multiplicity of wafers from a crysal composed of semiconductor material // Kaeser; Maximilian, Blank; Albert (DE); Assignee: Siltonic AG (DE).Appl. № 13/009957; Filed: 20.01.11.

4. S.P. Timoshenkov, V.V. Kalugin, Ye.P. Prokopiev, Chemical processing the surface of semiconductor wafers in the course of manufacturing KNI and micro-electronic facilities // Khimicheskaya tekhnologiya, No 12. p. 3-11 (2004), in Russian.

5. Yu. S. Zharkikh, S.V. Lisochenko, S.S. Novikov, O.V. Tretyak, Controlling the state of silicon wafer surface after chemical processing // Novyye tekhnologii, No 1-2, p. 18-20 (2004), in Russian.

6. John Zabasajja, Tushar Merchant, Belinda $\mathrm{Ng}$, Suman Banerjee, David Green, Modeling and characterization of tungsten chemical and mechenical polishing processes // J. Electrochem. Soc. 148(2), p. G73-G77 (2001).

7. V.N. Tomashik, Z.F. Tomashik, A.V. Lyubchenko, A.V. Fomin, Liquid-phase etching the semiconductor compounds of $\mathrm{A}^{\mathrm{II}} \mathrm{B}^{\mathrm{IV}}$ type and physico-chemical processes at their interfaces (Review) // Optoelektronika i poluprovodnikovaya tekhnika, No 28, p. 3-15 (1994), in Russian.

8. Z.F. Tomashik, V.N. Tomashik. Physico-chemical interaction of semiconductors of $\mathrm{A}^{\mathrm{II}} \mathrm{B}^{\mathrm{IV}}$ and $\mathrm{A}^{\mathrm{III}} \mathrm{B}^{\mathrm{V}}$ types with liquid etching components // Kondensirovannyye sredy $i$ mezhaznyye granitsy. No 4, p. 336-341 (2002), in Russian.

9. N.N. Grigoriyev, M.Yu. Kravetskyi, G.A. Pashchenko, S.A. Sypko, A.V. Fomin, Modelling the processes of contactless chemical-and-mechanical manufacturing the semiconductor sub-strates // Tekhnologiya $i$ konstruirovaniye $v$ electronnoi apparature, No 2, p. 36-40 (2003), in Russian.

10. N.N. Grigoriyev, M.Yu. Kravetskyi, G.A. Pashchenko, S.A. Sypko, A.V. Fomin, Kinetic characteristics of the process of contactless chemical-and-mechanical polishing the wafers // Fizicheskaya $i$ khimicheskaya obrabotka materialov, No 2, p. 61-67 (2004), in Russian. 